\title{
Ausser Atem - A couper le souffle
}

\section{Hansjakob Müller}

Prof. em. Dr. med., Medizinische Genetik, Universitätsspital Basel

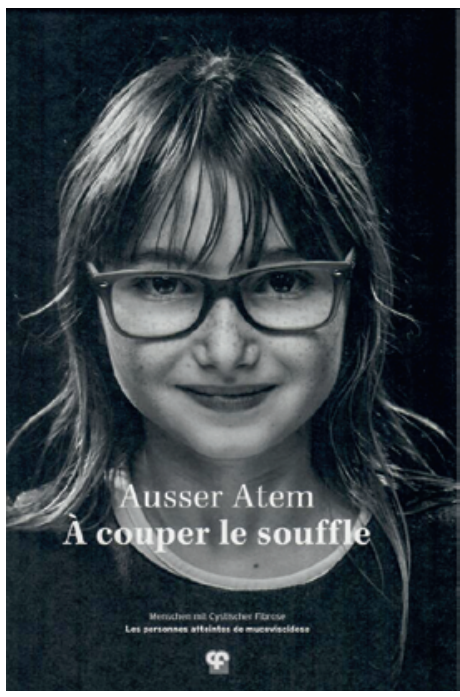

Ausser Atem - A couper le souffle

Herausgeber: Schweizerische Gesellschaft für Cystische Fibrose $\mathrm{CFCH}$, Altenbergstrasse 29, Postfach 686, 3000 Bern 8

$35 \mathrm{CHF}$

ISBN: 978-3-033-05749-4

Es geht hier nicht um den berühmten Gangsterfilm von Jean-Luc Godard aus dem Jahr 1960, sondern um ein eben erschienenes Buch mit gleichzeitig in deutscher und französischer Sprache verfassten Porträts von zwölf von der cystischen Fibrose (CF) Betroffenen,

\section{Die Porträts strahlen allgemein viel Zuversicht und Lebenskraft aus.}

in denen offen vom Leben mit dieser Erbkrankheit, von den damit verbundenen gesundheitlichen Problemen, dem zeitintensiven Therapieaufwand inklusive der Lungentransplantation, den Rückschlägen, den Hoffnungen und Träumen, aber auch über seelische Tiefs berichtet wird. Das Buch wurde von der Schweizerischen Gesellschaft für Cystische Fibrose zu ihrem 50-Jahr-Jubiläum herausgegeben.

Die Porträts strahlen allgemein viel Zuversicht und Lebenskraft aus. Die CF wird als Lebensaufgabe gesehen.
Aber auch psychische Belastungen (z.B. wegen begrenzter Lebenserwartung), Frustrationen (z.B. bei der Familienplanung) oder soziale Probleme (z.B. Trennung der Eltern) werden aufgezeigt. Erbkrankheiten bereiten begreiflicherweise Ängste und Sorgen, für die sich niemand zu schämen braucht, die nicht unterdrückt werden dürfen.

Ausser Atem will Eltern helfen, die eben erfahren haben, dass ihr Kind an der CF erkrankt ist, die CF-Patienten ihrerseits aber auch spüren lassen, dass sie mit ihrer Krankheit nicht alleine sind, und zudem das Verständnis für diese Erbkrankheit in der Öffentlichkeit und der Politik wecken und fördern.

Im Herbst 2014 hat der Bundesrat das Nationale Konzept für Menschen mit seltenen Krankheiten, zu denen die monogenen Erbkrankheiten gehören, verabschiedet; Betroffene sollen in Zukunft besser medizinisch betreut werden und eine wirkungsvollere Unterstüt-

Das Buch eignet sich ganz besonders als Lektüre für Personen, die sich in der Ausbildung zu einem Medizinalberuf befinden.

zung erhalten. Diesbezügliche Verbesserungen können nicht einfach top-down verordnet werden. Patientennähe ist beim Erkennen der jeweiligen Bedürfnisse und zur erfolgversprechenden Umsetzung von neuen Versorgungsstrukturen gefragt. Von den Betroffenen kann man Konkretes über das Leben mit einer bestimmten Erbkrankheit und über die dadurch ausgelösten alltäglichen Probleme erfahren.

Das Buch eignet sich ganz besonders als Lektüre für Personen, die sich in der Ausbildung zu einem Medizinalberuf befinden. Für den guten Umgang mit Patienten mit Erbkrankheiten ist zweifelsohne das Wissen über die dazu prädisponierenden Veränderungen $(\mathrm{Mu}-$ tationen) im Erbgut und die daraus resultierenden klinischen Auswirkungen wichtig. Letztlich handelt es sich aber immer um Mitmenschen mit ihren individuellen Eigenschaften, die in unterschiedlichen Situationen leben, die gut betreut sein wollen. Dafür gibt dieses Buch den CF-Patienten eine wichtige Stimme. 


\section{Weitere Bücher mit Erfahrungsberichten über das Leben mit einer Erbkrankheit}

\section{Hämophilie}

Arnold Gredig: Mein Leben mit 3H: Hämophilie - HIV - Hepatitis. Projekte-Verlag Cornelius GmbH Halle (2008), ISBN 978-3-86634-504-1 Kommentar: Sehr persönlicher Bericht eines Patienten über seine Erfahrungen als Bluter sowie als HIV- und Hepatitis-Infizierter.

\section{Marfan-Syndrom}

Marfan Stiftung Schweiz (Hrsg.): Herzsache. Gesundheitskompetenz und Empowerment bei chronischen körperlichen Beeinträchtigungen am Beispiel des Marfan-Syndroms. Marfan Stiftung Schweiz (2008), ISBN 978-3-033-01587-6

Kommentar: Aufgrund authentischer Beispiele wird gezeigt, wie erfolgreich mit einer zunehmenden körperlichen Beeinträchtigung umgegangen werden kann.

\section{Amyotrophe Lateralsklerose}

Gerhard Jenzer, Sonja Balmer: In der Überlebensfalle. Erfahrungen einer ALS-Patientin. Schwabe AG, Verlag, Basel (2008), ISBN 978-3-7965-2443-1

Kommentar: Das Buch geht auf Aufzeichnungen von Gesprächen zurück, die eine Patientin mit ihrem Neurologen führte. Zudem enthält es Informationen über die Krankheit aus der Sicht des Arztes.

\section{Von-Hippel-Lindau-Erkrankung und andere Erbkrankheiten} Hansjakob Müller und VHL-Schweiz (Hrsg.): Mit einer Erbkrankheit leben! Ein Ratgeber - auch für Jugendliche. EMH Schweizerischer Ärzteverlag AG, Basel (2014), ISBN 978-3-03754-078-7 Kommentar: Der kleine Ratgeber enthält praktische Hinweise von Fachleuten aus verschiedenen Fachgebieten sowie Erfahrungsberichte von mehreren Betroffenen mit verschiedenen Erbkrankheiten.

\section{Muskelkrankheiten (verschiedene)}

Helga Kessler: Starke Leben. Wie Muskelkranke ihren Alltag bewältigen. rüffer\&rub Sachbuchverlag, Zürich (2009), ISBN 978-3907625-46-0

Kommentar: Eine Journalistin zeigt in eindrücklichen Porträts auf, was muskelkranke Menschen bewegt, wie sie ihr Leben meistern. Im Anhang werden die Krankheiten der interviewten Patientinnen/Patienten vorgestellt.

\section{Hereditärer Brust- und Eierstrockkrebs}

Nicole Bürki, Denise C. Hürlimann, Ruth Baumann, Hansjakob Müller (Hrsg.): Familiärer Brustkrebs. Diagnose, Beratung, Therapie und Langzeitbetreuung. Schulthess Juristische Medien AG, Zürich (2012), ISBN 978-3-7255-6607-5

Kommentar: Neben zahlreichen gut verständlich abgefassten Beiträgen von Expertinnen/Experten der in der Betreuung involvierten Spezialgebiete enthält das Buch Erfahrungsberichte von betroffenen Frauen.

\section{Morbus Wilson}

M. D. Brower: Morbus Wilson - Ein Ratgeber für Patienten und

Demand (2006), ISBN 978-3-8334-4540-8

Kommentar: Das Buch gibt Patienten und der betreuenden Ärzteschaft einen umfassenden Überblick über Diagnose undTherapie der Kupferspeicherkrankheit.

\section{Kupfer-Kaleidoskop - Erfahrungsberichte}

Gesine Milde: Kupfer-Kaleidoskop - Erfahrungsberichte. Books on Demand (2009), ISBN 978-3-8391-0881-9

Kommentar: Es handelt sich um eine Sammlung von Erfahrungsberichten Betroffener über Symptomatik, Diagnostik und Therapie vom Morbus Wilson.

\section{Cystische Fibrose (Mukoviszidose)}

Manfred Ballmann, Reinald Fischer, Helge Hebestreit: Zystische Fibrose. Springer-Verlag GmbH (2014), ISBN 978-3-642-54319-7 Kommentar: Eine Sammlung von Patientenbeschreibungen aus der Praxis, die zeigt, welche Veränderungen des Allgemein- und Ernährungszustandes unter verschiedenen Voraussetzungen erreicht werden konnten.

Silvia Käppeli: "Man muss sich immer wieder neu einstellen». Mit cystischer Fibrose leben. Schriftenreihe der SGGP 119 (2013), ISBN 978-3-85707-119-5

Kommentar: Der Inhalt des Buches beruht auf Gesprächen mit CF-Patientinnen/-Patienten in der Schweiz. Es ist eine Quelle für Informationen, wie sie ihr Leben mit dieser fortschreitenden Krankheit erleben und wie sie mit einer Lungentransplantation umgehen.

\section{Prader-Willi-Syndrom}

Urs Eiholzer: Das Prader-Willi-Syndrom. Über den Umgang mit Betroffenen. S. Karger AG, Basel (2005), ISBN 3-8055-7845-8 Kommentar: Ein auf die Bedürfnisse des Alltags ausgerichteter Ratgeber, der sich an die Familienangehörigen, aber auch an alle anderen Personen richtet, die mit betroffenen Kindern in Kontakt kommen.

Urs und Susanne Stöckli: «Unterwegs mit Manuel». 16 Briefe an Freunde und Angehörige. 2. Auflage (2007), erhältlich bei der Prader-Willi-Syndrom Vereinigung Schweiz (www.prader-willi.ch) Kommentar: Es handelt sich um Briefe an Freunde und Angehörige einer Pfarrfamilie mit einem betroffenen Kind, die es erlauben, an ihrem Schicksal teilzuhaben. Sie geben einen un mittelbaren Einblick zu vielen mit dem Prader-Willi-Syndrom verbundenen medizinischen, aber auch psychosozialen Fragen, aber auch Antworten dazu!

\section{Leukodystrophie}

Anne-Dauphine Julliand: Deine Schritte im Sand. Das kurze, aber glückliche Leben meiner Tochter. Lübbe Digital (2012), ISBN 978-3-83871-953-3 (aus dem Französischen übersetzt)

Kommentar: Anne-Dauphine Julliand berichtet vom Leben mit ihrerTochterThais, die an einer früh im Leben sich manifestierenden Form dieser Gruppe von Erbkrankheiten leidet, und von ihrem Umgang mit deren Tod.

Fiktionale Auseinandersetzungen mit einer Erbkrankheit Beate Rothmaier: Atmen, bis die Flut kommt. Roman. DVA (2013), ISBN 978-3-421-04495-2

Kommentar: Die Schriftstellerin berichtet sprachlich gewandt über das Leben als Alleinerziehende eines behinderten Kindes. Sie überträgt diese Rolle mit allen Auflehnungen und Verantwortungen dem Vater, einem Comiczeichner.
Anmerkung: Diese Bücherliste erhebt keinen Anspruch auf Vollständigkeit. 